\title{
Perturbation-Iteration Method for Solving Mathematical Model of Blood Partial Pressures in Human Cardiovascular-Respiratory System during Physical Activity
}

\author{
Ntaganda Jean Marie \\ Department of Mathematics \\ School of Science \\ College of Science and Technology \\ University of Rwanda \\ Kigali, Rwanda
}

\begin{abstract}
This paper aims at solving of a two ordinary differential models equations for cardiovascularrespiratory system using a new recent method: Perturbation Iteration method. The description of this method for different orders of derivative in the Taylor Series expansion is discussed. This method provides the solution in the form of an infinite series for ordinary differential equation. The efficiency of the method used is investigated by a comparison of Euler method and Runge Kutta. The numerical simulations of all theses three methods are implemented in Matlab. The validation has been carried out by taking the values of determinant parameters of cardiovascular-respiratory system for a 30 years old woman who is supposed to do three in regular physical activity: Walking, Jogging and Running fast. The results are in good agreement with experimental data.
\end{abstract}

Keywords: Perturbation iteration method, Cardiovascular/respiratory system, Heart rate, Alveolar ventilation, Systemic arterial pressure, Systemic venous pressure, Numerical simulation.

\section{INTRODUCTION}

The behaviour of cardiovascular and metabolic disorders depend on the complex interplay of multiple anatomic and physiologic factors but the mechanism behind these factors and their impacts on the type or degree of the disorder, experimental observations or on the treatment responses remain poorly understood. Therefore, most patients today are being treated with general therapies regardless of the cause of dysfunction. We believe that combining experimental measurements with mathematical modeling provides an important information on the individual key dysfunction, making it possible to start developing personalized therapies. Similarly as observations from data can inspire new theoretical models, the models can translate the measurements first into new ideas, then into testable hypotheses and finally into medical knowledge. Such model-based investigations can therefore provide systematic strategies toward better understanding, predicting or even preventing the disorders. The aim of the modeling work is to use differential equations to describe the complex mechanisms of the cardiovascular-respiratory control system. The mathematical model equations contains a certain number of parameters to be estimated. The possibility of estimating them is use a simple, noninvasive measurement techniques that would be of great clinical importance for better understanding the physiologic abnormalities underlying various disease conditions, including cardiovascular and metabolic diseases. In this way, the models could be used for translating the hidden information from the experimental measurements into physiologically meaningful individual parameters, providing novel features for diagnostic prediction and treatment outcomes when developing new therapies.

Since 1950's, it has been developed a number of dynamic models of the human cardio-respiratory system. Most of them arise from the compartmental theory [1, 2, 3, 4]. The modeling approach done by Timischl [3] has been opportunity to show the role of physical activity in controlling cardiovascular-respiratory system. It shows also the role of pulmonary circulatory resistance during exercise. Based on existing global models of cardiovascular and respiratory system, the design of a 
new mathematical model that provides global solutions by the use of inverse processes has been presented in [5]. The results obtained show that it possible to find approximately an optimal way to maintain blood partial pressures to its desired values that are clinically accepted. The numerical resolution of mathematical models of cardiovascular-respiratory system can be done using different numerical methods. The recent method that can be used has been derived in [6]: Perturbation Iteration method. This paper focuses on this new important method.

This paper is organised as follows. In section 2, we present the mathematical model of ordinary differential equations. The section 3 deals with the the basic idea of the Perturbation Iteration method. The numerical simulation is presented in section 4 where the comparison is done using Euler method and Runge Kutta method to test efficiency of perturbation iteration method. The section 5 focuses on discussion while Section 6 rounds up and deals with the concluding remarks.

\section{The Mathematical Model EQuations}

The cardiovascular and respiratory system plays a crucial role in controlling the blood flow of human body. The main controls of pressures are heart rate and alveolar ventilation which control the systemic arterial pressures to prevent cardiac accidents [7]. For a healthy subject, it is well known that heart rate and alveolar ventilation depend on his/her level in training during physical activity. A global mathematical model for physical activity developed by S. Timischl-Teschl [8] shows the instability of equilibrium steady. It is governed by many differential equations and it doesn't permit to understand a long-term cardiovascular- respiratory system in the case of aerobic physical activities. A two compartmental mathematical model has been developed in [5] to solve this problem. We consider the functions $P_{v s}$ and $P_{a s}$ as respectively mean blood pressures in systemic arterial region and in systemic venous region. $H$ and $\dot{V}_{A}$ denote the control functions. For a cardiovascular- respiratory system, they design heart rate and alveolar ventilation respectively. The mechanism of this control is not direct and can be represented by outflow functions between systemic arterial and venous compartments that depend on heart rate and alveolar ventilation. The equations model arise from straightforward development of mass balance between arterial systemic arterial and systemic venous compartments. For three physical activities (Walking, Jogging and Running fast), the ODE mathematical model is

$$
\left\{\begin{array}{l}
\frac{d P_{a s}(t)}{d t}=-P_{a s}(t)+P_{v s}^{\gamma}(t) f\left(H, \dot{V}_{A}\right) \\
\frac{d P_{v s}(t)}{d t}=-P_{v s}(t)+P_{a s}^{\delta}(t) g\left(H, \dot{V}_{A}\right) \\
P_{a s}(0)=P_{a s}^{0}, \quad P_{v s}(0)=P_{v s}^{0},
\end{array}\right.
$$

where the constants of the model equations (1) are given as $\gamma=-0.0112, \delta=-0.1724$ and the functions $f$ and $g$ have been identified as follows [5].

1) Walking case:

$$
\begin{aligned}
& f\left(H, \dot{V}_{A}\right)=\exp \left(2.6812 \dot{V}_{A}^{-0.0479}+3.4921 H^{-0.0943}\right), \\
& g\left(H, \dot{V}_{A}\right)=\dot{V}_{A} \exp \left(H^{-30.7207}-0.0881\right)
\end{aligned}
$$

2) Jogging case:

$$
\begin{aligned}
& f\left(H, \dot{V}_{A}\right)=\exp \left(0.9990 \dot{V}_{A}^{0.1179}+1.1522 H^{0.2280}\right), \\
& g\left(H, \dot{V}_{A}\right)=\dot{V}_{A} \exp \left(H^{-0.3245}-0.8699\right)
\end{aligned}
$$

3) Running fast:

$$
\begin{aligned}
& f\left(H, \dot{V}_{A}\right)=\exp \quad\left(0.5472 \dot{V}_{A}^{0.3820}+0.7518 H^{0.2869}\right), \\
& g\left(H, \dot{V}_{A}\right)=\dot{V}_{A} \exp \quad\left(H^{-0.0992}-1.7521\right) .
\end{aligned}
$$


Taking $H_{e}$ and $\dot{V}_{A_{e}}$ heart rate and alveolar ventilation at the equilibrium states, the system (1) is written as

$$
\left\{\begin{array}{l}
\frac{d P_{a s}(t)}{d t}=-P_{a s}(t)+P_{v s}^{\gamma}(t) f_{e} \\
\frac{d P_{v s}(t)}{d t}=-P_{v s}(t)+P_{a s}^{\delta}(t) g_{e} \\
P_{a s}(0)=P_{a s}^{0}, \quad P_{v s}(0)=P_{v s}^{0},
\end{array}\right.
$$

where where $f_{e}=f\left(H_{e}, \dot{V}_{A_{e}}\right)$ and $g_{e}=g\left(H_{e}, \dot{V}_{A_{e}}\right)$.

\section{Perturbation Iteration Algorithm (PIA)}

Perturbation-iteration method has been developed recently by Aksoy and al. [6]. This new method of solving a system of first order of nonlinear ordinary differential equations uses a combination of perturbation expansions and Taylor series expansions to give rise to an iteration scheme where Aksoy and al. [6] and Pakdemirli [9] introduced expansion and correction terms of only first derivatives in the Taylor series expansion and one correction term in the perturbation. Therefore, the Perturbationiteration algorithm is named by $P I A(1,1)$. Let us be interested in the description of $P I A(1,1)$.

First of all, we discuss the PIA $(1, \mathrm{~m})$ which is constructed by taking one correction term in the perturbation expansion and correction terms of $\mathrm{m}^{\text {th }}$ order derivatives in the Taylor Series expansion. We consider a system of first order of $K$ nonlinear ordinary differential equations. We note

$$
x=\left(x_{1}, x_{2}, \ldots, x_{K}\right)^{T}
$$

a vector state. The system first order of $K$ nonlinear ordinary differential equations can be written as follows

$$
E_{k} \equiv E_{k}\left(\dot{x}_{k}, x_{j}, \varepsilon, t\right)=0, \quad k=1,2, \ldots, K, j=1,2, \ldots, K
$$

where $\varepsilon$ is the perturbation parameter and $t$ denotes the independent variable. That is the system

$$
\left\{\begin{array}{c}
E_{1} \equiv\left(\dot{x}_{1}, x_{1}, x_{2}, \ldots, x_{K}, \varepsilon, t\right)=0 \\
E_{2} \equiv\left(\dot{x}_{2}, x_{1}, x_{2}, \ldots, x_{K}, \varepsilon, t\right)=0 \\
\vdots \\
E_{K} \equiv\left(\dot{x}_{k}, x_{1}, x_{2}, \ldots, x_{K}, \varepsilon, t\right)=0 .
\end{array}\right.
$$

Taking an approximate solution of the system (3) as

$$
x_{k, n+1}=x_{k, n}+\varepsilon x_{k, n}^{c}
$$

where subscript $n$ represents the $\mathrm{n}^{\text {th }}$ iteration over this approximate solution, we have a solution with one correction term in the perturbation expansion. The system can be approximated with a Taylor series expansion in the neighborhood of $\varepsilon=0$

$$
E_{k}=\sum_{i=0}^{m} \frac{1}{i !}\left[\left(\frac{d}{d \varepsilon}\right)^{i} E_{k}\right]_{\varepsilon=0} \varepsilon^{i}, \quad k=1,2, \ldots, K
$$

where

$$
\frac{d}{d \varepsilon}=\frac{\partial \dot{x}_{k, n+1}}{\partial \varepsilon} \frac{\partial}{\partial \dot{x}_{k, n+1}}+\left(\sum_{j=0}^{K} \frac{\partial x_{j, n+1}}{\partial \varepsilon} \frac{\partial}{\partial x_{j, n+1}}\right)+\frac{\partial}{\partial \varepsilon}
$$

is defined for the $(\mathrm{n}+1)^{\text {th }}$ iterative equation 


$$
E_{k}\left(\dot{x}_{k, n+1}, x_{j, n+1}, \varepsilon, t\right) \text {. }
$$

Substituting (6) into (5), we obtain an iteration equation

$$
E_{k}=\sum_{i=0}^{m} \frac{1}{i !}\left[\left(\dot{x}_{k, n}^{c} \frac{\partial}{\partial \dot{x}_{k, n+1}}+\left(\sum_{j=0}^{K} x_{j, n}^{c} \frac{\partial}{\partial x_{j, n+1}}\right)+\frac{\partial}{\partial \varepsilon}\right)^{i} E_{k}\right]_{\varepsilon=0} \varepsilon^{i}, \quad k=1,2, \ldots, K
$$

which is a first order differential equation and can be solved for the correction terms $x_{k, n}^{c}$. Then using $(4)$, the $(n+1)^{\text {th }}$ iteration solution can be found. Iterations are terminated after a successful approximation is obtained.

Note that for a more general algorithm, $\mathrm{n}$ correction terms instead of one can be taken in expansion (4) which would then be a $\operatorname{PIA}(n, m)$ algorithm. The algorithm can also be generalized to a differential equation system having arbitrary order of derivatives (See [10] for more details).

After the discussion of $\operatorname{PIA}(1, m)$, now we focus on $\operatorname{PIA}(1,1)$ which is its simple case of perturbation-iteration method $\operatorname{PIA}(n, m)$.

We consider the general cause of first order of differential equation as

$E(\dot{x}, x, \varepsilon)=0$

where $x=x(t)$. Taking one correction term in perturbation expansion, we have

$$
x_{n+1}=x_{n}+\varepsilon x^{c}
$$

where $n$ denotes the nth iteration over this approximate solution such that for the perturbation parameter $\varepsilon$ the expression $\varepsilon x^{c}$ represents the correction term. Substitution of (9) into (8) we obtain

$$
E\left(\dot{x}_{n}, x_{n}, 0\right)+\frac{\partial E(\dot{x}, x, 0)}{\partial x} \varepsilon x^{c}+\frac{\partial E\left(\dot{x}_{n}, x_{n}, 0\right)}{\partial \dot{x}} \varepsilon \dot{x}^{c}+\frac{\partial E\left(\dot{x}_{n}, x_{n}, 0\right)}{\partial \varepsilon} \varepsilon=0
$$

Reorganizing the equation (10), we have

$$
\dot{x}^{c}+\frac{E_{x}}{E_{\dot{x}}} x^{c}=-\frac{\varepsilon E_{\varepsilon}+E}{\varepsilon E_{\dot{x}}}
$$

where $E_{z}=\frac{\partial E}{\partial z}$ and all derivatives are evaluated at $\varepsilon=0$. Setting

$$
\mu(t)=\exp \left(\int \frac{E_{x}}{E_{\dot{x}}} d t\right)
$$

as integrating factor, our equation (11) is now transformed into the form

$$
\frac{d}{d t}\left(\mu(t) x^{c}\right)=\mu(t)\left(-\frac{\varepsilon E_{\varepsilon}+E}{\varepsilon E_{\dot{x}}}\right)
$$

so that

$$
\mu(t) x^{c}=-\int \mu(t)\left(\frac{\varepsilon E_{\varepsilon}+E}{\varepsilon E_{\dot{x}}}\right)+C
$$

that is 


$$
\begin{aligned}
x^{c} & =\frac{C}{\mu(t)}-\frac{1}{\mu(t)} \int \mu(t)\left(\frac{\varepsilon E_{\varepsilon}+E}{\varepsilon E_{\dot{x}}}\right) \\
& =C \exp \left(-\int \frac{E_{x}}{E_{\dot{x}}} d t\right)-\left[\int\left(\frac{\varepsilon E_{\varepsilon}+E}{\varepsilon E_{\dot{x}}}\right) \exp \left(\int \frac{E_{x}}{E_{\dot{x}}} d t\right)\right] \exp \left(-\int \frac{E_{x}}{E_{\dot{x}}} d t\right)
\end{aligned}
$$

Substitution of (12) into (9) and constructing the iteration scheme yields

$$
\begin{aligned}
x_{n+1}=x_{n}+\varepsilon C_{n} C & \exp \left(-\int \frac{E_{x}\left(\dot{x}_{n}, x_{n}, 0\right)}{E_{\dot{x}}\left(\dot{x}_{n}, x_{n}, 0\right)} d t\right)-\left[\int\left(\frac{\varepsilon E_{\varepsilon}\left(\dot{x}_{n}, x_{n}, 0\right)+E\left(\dot{x}_{n}, x_{n}, 0\right)}{\varepsilon E_{\dot{x}}\left(\dot{x}_{n}, x_{n}, 0\right)}\right)\right. \\
& \left.\exp \left(\int \frac{E_{x}\left(\dot{x}_{n}, x_{n}, 0\right)}{E_{\dot{x}}\left(\dot{x}_{n}, x_{n}, 0\right)} d t\right)\right] \exp \left(-\int \frac{E_{x}\left(\dot{x}_{n}, x_{n}, 0\right)}{E_{\dot{x}}\left(\dot{x}_{n}, x_{n}, 0\right)} d t\right) .
\end{aligned}
$$

\section{Numerical Simulation}

To show the effectiveness of PIA(1,1) , we focus on the numerical simulation of the system () by taking the observed values presented in the table 1 [5] for three case of physical activities that is walking, jogging, running fast.

Table 1: The mean value of the heart rate, the alveolar ventilation, venous and arterial systemic pressure for the rest and three cases of physical activities. A part the rest, other numbers represent equilibrium values related to 30 years old woman three physical activities. A part the rest, other numbers represent equilibrium values related to 30 years old woman three physical activity.

\begin{tabular}{|l|l|l|l|l|}
\hline Exercise intensity & Rest & Walking & Jogging & Running Fast \\
\hline Ventilation $(\mathrm{L} / \mathrm{min})$ & 6 & 8.5 & 15 & 25 \\
\hline Heart rate $(\mathrm{Beats} / \mathrm{min})$ & 70 & 85 & 140 & 180 \\
\hline Arterial Pas $(\mathrm{mmHg})$ & 104 & 110 & 135 & 170 \\
\hline Venous Pvs(mmHg) & 3.566 & 3.46 & 3.28 & 3.23 \\
\hline
\end{tabular}

Taking $P_{a s}(0)=104$ and $P_{v s}(0)=3.566$ as given, the system (2) is solved by using $\operatorname{PIA}(1,1)$.

The perturbation parameter $F$ is artificially introduced as

$\left\{\begin{array}{l}E_{1} \equiv \dot{P}_{a s}+P_{a s}-\varepsilon P_{v s}^{\gamma} f_{e}=0 \\ E_{2} \equiv \dot{P}_{v s}+P_{v s}-\varepsilon P_{a s}^{\delta} g_{e}=0 .\end{array}\right.$

Setting $X=\left(P_{a s}, P_{v s}\right)^{T}$, we want an approximate solution of the system of the form

$$
X_{k, n+1}=X_{k, n}+\varepsilon X_{k, n}^{c}, k=1,2 \text {. }
$$

with one correction term in the perturbation expansion such that the relation () is satisfied for

$E_{k}\left(\dot{X}_{k, n+1}, X_{k, n+1}, \varepsilon\right), \quad k=1,2 ; \quad n=0,1,2, \ldots$

For (13), equation (14) reduces to

$$
\left\{\begin{array}{l}
E_{1} \equiv \varepsilon \dot{X}_{1, n+1}^{c}+\varepsilon X_{1, n}^{c}=-\dot{X}_{1, n}-X_{1, n}+f_{e} X_{2, n}^{\gamma} \\
E_{2} \equiv \varepsilon \dot{X}_{2, n+1}^{c}+\varepsilon X_{2, n}^{c}=-\dot{X}_{2, n}-X_{2, n}+g_{e} X_{1, n}^{\delta} .
\end{array}\right.
$$

with the solution

$$
X_{1, n}^{c}=\frac{e^{-t}}{\varepsilon}\left[\int\left(-\dot{X}_{1, n}-X_{1, n}+f_{e} X_{2, n}^{\gamma}\right) e^{t} d t+C_{1, n}\right]
$$

and 


$$
X_{1, n}^{c}=\frac{e^{-t}}{\varepsilon}\left[\int\left(-\dot{X}_{2, n}-X_{2, n}+g_{e} X_{1, n}^{\delta}\right) e^{t} d t+C_{2, n}\right]
$$

where $C_{1, n}$ and $C_{2, n}$ are constants of integration that have to be determined at each iteration from initial guess satisfying the initial condition if (14) is applied.

Using the values from the table 1 , we obtain the values of $f_{e}$ and $g_{e}$ as shown the table 2 for three cases of physical activity.

Table 2. The values $f_{e}$ and $g_{e}$ for three cases of physical activity.

\begin{tabular}{|l|l|l|l|}
\hline Function $f$ and g at steady state & Walking & Jogging & Running Fast \\
\hline $\mathrm{f}_{\mathrm{e}}$ & 111.818 & 138.3524 & 182.4921 \\
\hline $\mathrm{g}_{\mathrm{e}}$ & 7.7057 & 7.6454 & 7.89951 \\
\hline
\end{tabular}

After substituting initial guess in (15) and with the help of equation (14), the first following approximation has been obtained in applying the iteration formula (16) and (17).

- Walking case:

$$
\begin{aligned}
& X_{1,1}=-6.23700 e^{-t}+110.2370 \\
& X_{2,1}=0.1072 e^{-t}+3.4588
\end{aligned}
$$

- Jogging case:

$$
\begin{aligned}
& X_{1,1}=-32.39620 e^{-t}+136.3962 \\
& X_{2,1}=0.2905 e^{-t}+3.2755
\end{aligned}
$$

- Running fast case:

$$
\begin{aligned}
& X_{1,1}=-75.9118 e^{-t}+179.9118 \\
& X_{2,1}=0.3355 e^{-t}+3.2305
\end{aligned}
$$

To test the efficiency of perturbation iteration algorithm PIA(1,1) we compare the results with two others obtained using two different methods which are Euler method and Matlab approach for solving system of ordinary differential equations (ODEs). The Matlab approach has been implemented using its ODEs solver from Runge-Kutta of order 4 and 5, this is ode45. The numerical results are illustrated in the figure 1,2 and 3.
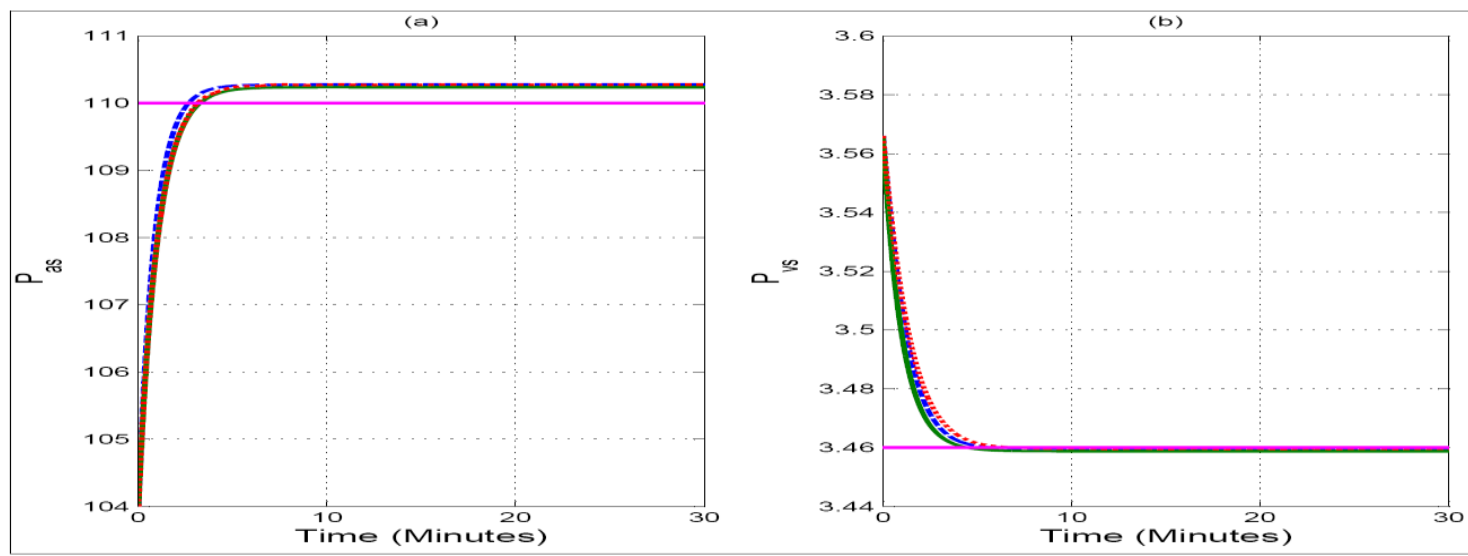

Figure 1. Variation trajectory of blood systemic arterial pressure, $P_{a s}(a)$ and blood systemic venous pressure, $P_{v s}(b)$ for a 30 years old woman during walking physical activity. Three curves are compared using three different methods: Perturbation iteration algorithm (Solid line), Euler method (dashed line) and Matlab approach (Dot line) 
Perturbation-Iteration Method for Solving Mathematical Model of Blood Partial Pressures in Human Cardiovascular-Respiratory System during Physical Activity

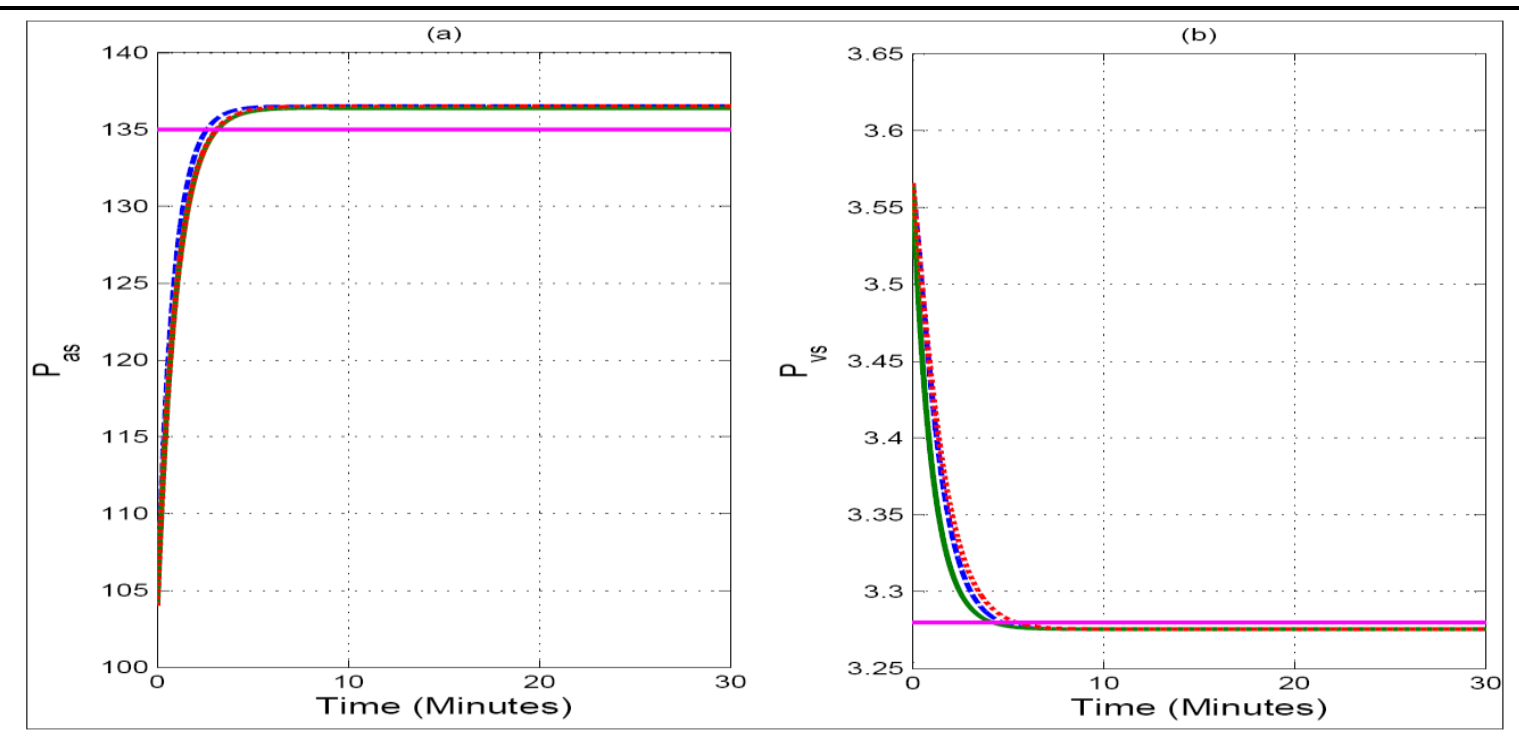

Figure 2: Variation trajectory of blood systemic arterial pressure, $P_{a s}(a)$ and blood systemic venous pressure, $P_{v s}(b)$ for a 30 years old woman during jogging physical activity. Three curves are compared using three different methods: Perturbation iteration algorithm (Solid line), Euler method (dashed line) and Matlab approach (Dot line).

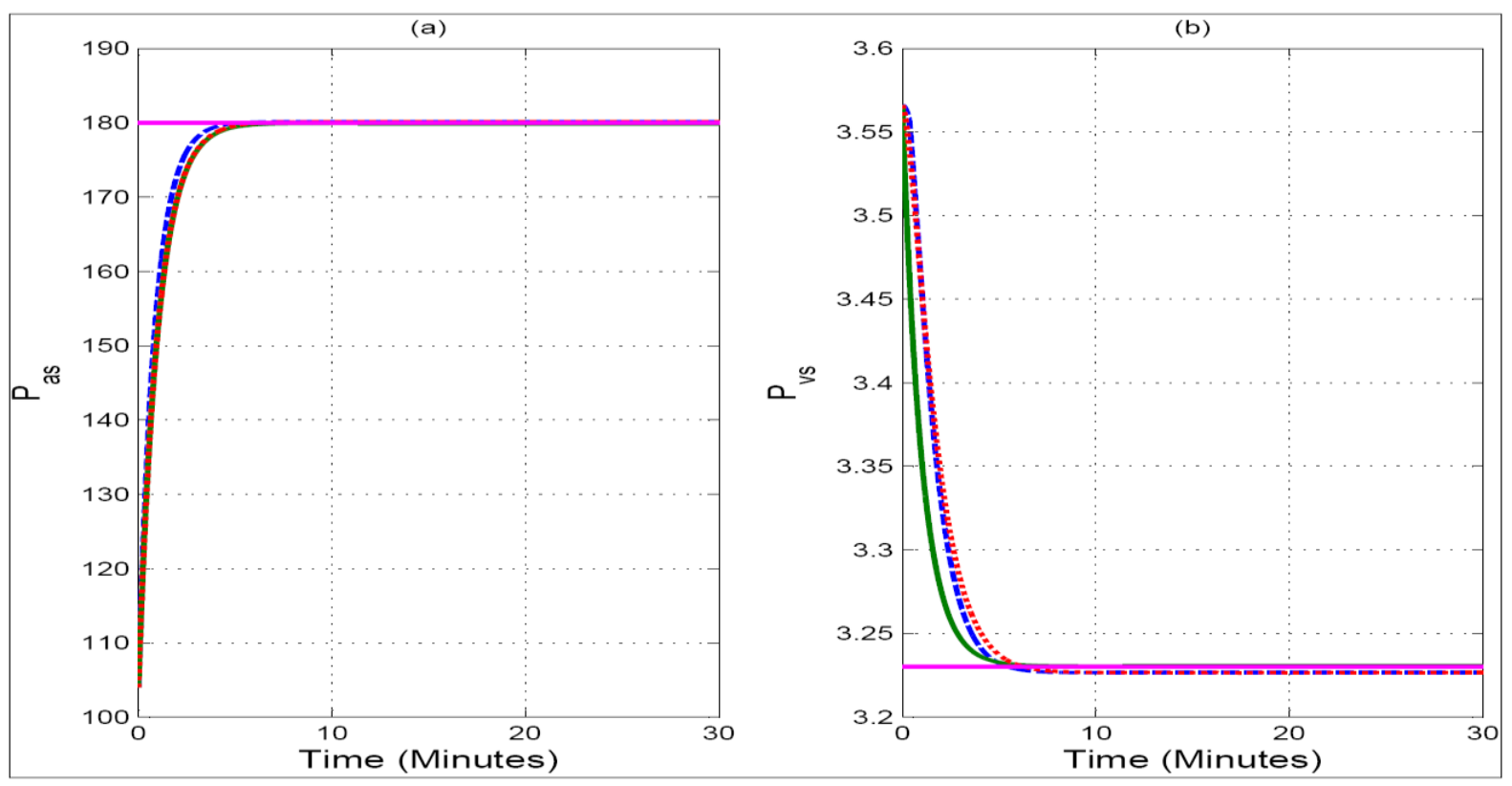

Figure 3. Variation trajectory of blood systemic arterial pressure, $P_{a s}(a)$ and blood systemic venous pressure, $P_{v s}(b)$ for a 30 years old woman during running fast physical activity. Three curves are compared using three different methods: Perturbation iteration algorithm (Solid line), Euler method (dashed line) and Matlab approach (Dot line)

\section{DisCuSSION}

The heart rate and the alveolar ventilation are two controls of the cardiovascular-respiratory system. The stability of each of them at the equilibrium value allows other determinant parameters of cardiovascular-respiratory system to reach a stabilized state. The response of these controls to the blood systemic arterial pressure and the blood systemic venous pressure are represented in figure 1, 2 and 3 where all methods used show that blood systemic arterial pressure increases at the beginning of physical activity and it is stabilized when it reaches the corresponding equilibrium value (See figure 1(a), 2(a) and 3(a). This shows a perfect representation of the importance of physical activity in the regulation of the cardiovascular respiratory system, particularly the increase of the blood systemic arterial pressure during exercise. The ventilation rate plays an important role in the gas supply and 
regulation through the body. An increase in heart rate and ventilation rate results in an adequate and regular supply of both oxygen and carbon dioxide in the body. The figures 1(b), 2(b) and 3(b) show a decrease of blood systemic venous pressure at the beginning of physical activity until is reaches the equilibrium value where it is stabilized. This results from the brut increase of ventilation during the initial stage of the physical activity which allows a gradual increase of blood systemic venous pressure. The results obtained in this work are rather satisfactory. In particular, the reaction of the cardiovascular-respiratory system to physical activity can be modelled and a feedback can be approximated by the solution of its mathematical model governed by ordinary differential system. Physical activity reduces any cardiovascular-respiratory system disease and it induces important changes in the stabilization of cardiac, vascular and blood tissue.

\section{Concluding Remarks}

We have investigated in this work a new numerical method for solving a system of ordinary differential equations: Perturbation Iteration method. The efficiency of this method is tested using two other convergent methods that is Euler method and Runge-Kutta method. Those all method are implemented using Matlab packages. The numerical simulations illustrate the responses of the blood systemic arterial pressure and blood systemic due to the controls heart rate and alveolar ventilation of cardiovascular-respiratory system. The numerical results confirmed the analytical analysis for a 30 years old woman during three different physical activities: Walking, Jogging and Running fast.

\section{REFERENCES}

[1] F. Kappel, and R. O. Peer, A mathematical model for fundamental regulation processes in the cardiovascular model, J. Math. Biol. 31(6), pp. 611- 631, 1993.

[2] F. Kappel, S. Lafer, and R. O. Peer, A model for the cardiovascular system under an ergometric workload, Surv. Math. Ind. 7, pp.239-250, 1997.

[3] S. Timischl, A global Model for the Cardiovascular and Respiratory System, $\mathrm{PhD}$ thesis, KarlFranzens-Universit of Graz, August 1998.

[4] S. Timischl-Teschl, Modeling the human Cardiovascular-Respiratory Control System: An optimal Control Application to the Transition to Non-REM Sleep, Mathematical Biosciences and engineering - Volume 7, Number 07, 2004.

[5] Jean Marie Ntaganda and Benjamin Mampassi, Modelling blood partial Pressures of the human cardiovascular respiratory system, Applied Mathematics and computation, Vol.187, pp 11001108, 2007.

[6] Aksoy Y. and Pakdemirli M. New perturbation-iteration solutions for Bratu-type equations, Computers and Mathematics with Applications, vol. 59, no. 8, pp.2802-2808, 2010.

[7] S. Sepehris, Physical model of human respiration, Young Researchers club, Islamic Azad university of Shiraz, 12-17, 2007.

[8] S. Timischl, A global Model for the Cardiovascular and Respiratory System, PhD thesis, KarlFranzens-Universit of Graz, August 1998.

[9] Pakdemirli M., Aksoy, Y. and Boyaci, H. A new perturbation-iteration approach for first order differential equations, Mathematical and Computational Applications, vol. 16, no. 4, pp.890-899, 2011.

[10] Pakdemirli M, Review of the perturbation-iteration method, Mathematical and Computational Applications, Vol. 18, No. 3, pp. 139-151, 2013

\section{AUTHOR's BIOGRAPHY}

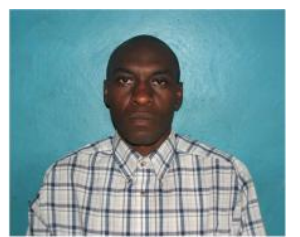

Prof. Ntaganda Jean Marie, PhD holder in Numerical Analysis from Cheikh Anta Diop University of Dakar, Senegal (2004-2006) and Ouagadougou University, Burkina Faso (2004-2007). He is Associate Professor in Applied Mathematics at University of Rwanda, College of Science and Technology, School of Sciences, Department of Mathematics where he is module leader of computation modules including Numerical Analysis and Programming. Since 2005 he is active researcher in Biomathematics where at least 35 publications have been published in international peer-reviewed journals. 